\title{
Violencia de género en tiempos de covid-19 en mujeres del programa vaso de leche del distrito de Huancavelica 2021
}

\author{
Gender violence in times of covid-19 in women from the vaso de leche program of the district of \\ Huancavelica 2021
}

María Del Carmen Valencia Poma ${ }^{1, a}$, Jenny Mendoza Vilcahuaman ${ }^{1, b}$

\section{RESUMEN}

Objetivo: Determinar los tipos de violencia de género que más predomino en tiempos de COVID-19 en mujeres del Programa Vaso de Leche (PVL) del Distrito de Huancavelica 2021. Material y Métodos: El estudio fue de tipo descriptivo, observacional, retrospectivo, transversal; con una población y muestra de 47 mujeres presidentas del PVL. Para la recolección de datos se utilizó un cuestionario, luego se procesaran en el programa estadístico SPSS versión 22. Resultados: De todas las entrevistadas (47); fueron víctimas de violencia psicológía $65.9 \%$, violencia económica 40,4\%, violencia física 31,9\% y violencia sexual $21,3 \%$. Acerca de las características sociodemográficas predominaron: Adultos $74,5 \%$, nivel secundario $48,9 \%$, conviviente $61,7 \%$, ama de casa $83,0 \%$ y zona urbana $63,8 \%$. Efectos de la violencia psicológica (42): Ansiedad y depresión $61,9 \%$ y baja autoestima $38,1 \%$; de la violencia física (15): Hematomas 46,7\% y lesiones, heridas 53,3\%; de la violencia sexual (10): Embarazo no deseado $20,0 \%$ y disminución del deseo sexual 80,0\%; de la violencia económica (31): Bajo ingreso económico 58,1\% y perdida de oportunidad educativa y laboral $41,9 \%$. Conclusión: Los tipos de violencia que más predominaron fueron la psicológica y económica; la mayoría de estas mujeres fueron adultas, de nivel secundaria, convivientes y de procedencia urbana. Las consecuencias fueron múltiples, lo cual resalta un grave problema en el bienestar de esta población.

PALABRAS CLAVE: Violencia de Género, violencia psicológica, violencia física, violencia sexual y violencia económica.

\section{SUMMARY}

Objective: To determine the types of gender violence that most predominated in times of COVID-19 in women of Vaso de Leche Program (PVL) from Huancavelica district 2021. Material and Methods: The study was descriptive, observational, retrospective, cross-sectional; with a population and sample of 47 women presidents of the PVL. For data collection, a questionnaire was used, then it was processed in the statistical program SPSS version 22. Results: Of all the interviewees (47); $65.9 \%$ were victims of psychological violence, $40.4 \%$ economic violence, $31.9 \%$ physical violence and $21.3 \%$ sexual violence. About the sociodemographic characteristics predominantly: Adults $74.5 \%$, secondary level $48.9 \%$, cohabiting $61.7 \%$, housewife $83.0 \%$ and urban area $63.8 \%$. Effects of psychological violence (42): Anxiety and depression $61.9 \%$ and low self-esteem $38.1 \%$; of physical violence (15):

\footnotetext{
Universidad Nacional De Huancavelica, Huancavelica, Perú.

Estudiante, ORCID ID: 0000-0003-2802-404X

Doctora. ORCID ID: 0000-0002-1903-1355
} 
bruises $46.7 \%$ and injuries, wounds $53.3 \%$; of sexual violence (10): unwanted pregnancy $20.0 \%$ and decrease in sexual desire $80.0 \%$; of economic violence (31): Low income $58.1 \%$ and loss of educational and work opportunity 41.9\%. Conclusions: The most prevalent types of violence were psychological and economic; Most of these women were adults, of secondary level, cohabiting and of urban origin. The consequences were multiple, which highlights a serious problem in the well-being of this population.

KEYWORDS: Gender violence, psychological violence, physical violence, sexual violence and economic violence.

\section{INTRODUCCIÓN}

La violencia de género o violencia contra la mujer es un fenómeno que afecta a todos los países sin distención de clases sociales, se presenta en las distintas etapas de vida, la cual afecta a la salud pública. Las Naciones Unidas definen a la violencia de género a "cualquier acto de violencia que tenga o que pueda tener como resultado un daño o sufrimiento físico, sexual o psicológico para la mujer, incluyendo las amenazas de dichos actos, la imposición o privación injusta de la libertad, ya sea en la vida pública o privada" (1).

Sin dejar de mencionar que la violencia contra la mujer es un problema de salud mundial de proporciones epidémicas. Según las estadísticas, indican que de cada tres mujeres una sufre de violencia de género, al menos el 35\% de mujeres en todo el mundo han sido víctimas de violencia física y/o sexual de la pareja o violencia sexual por terceros, esto en algún momento de su vida $(2,3)$.

A causa de la pandemia de la COVID-19 se ha observado un aumento violento del porcentaje de los casos de violencia de género, no solo a nivel local, sino que también a nivel nacional y mundial, ya que la coyuntura hace que la mujer pase mayor tiempo en su hogar junto a su agresor y por ende esté en peligro (4). En el país la violencia contra la mujer se ha realizado desde tiempos muy remotos, por tal motivo muchas organizaciones e instituciones luchan por tratar de reducir este problema, sin embargo, en la actualidad la cifra de víctimas se ha elevado a causa de la pandemia, ya que dichas instituciones no estuvieron preparadas para esta coyuntura. El Ministerio de la Mujer y Poblaciones Vulnerables (MIMP) registraron un total de 67,712 casos de violencia familiar y abuso sexual de los cuales 17,000 de las víctimas fueron entre niñas, niños y adolescentes, Asimismo, los tipos de violencia muestran un $11 \%$ en violencia sexual, $40 \%$ en violencia física y $49 \%$ en violencia psicológica. Dichos casos se atendieron mediante la Línea 100 del MIMP durante los 107 primeros días desde que empezó cuarentena general (5).
En Huancavelica, la Defensoría del Pueblo junto con el Programa Aurora y los 9 Centros de Emergencia Mujer, atendieron un total de 706 casos de violencia desde que empezó la cuarentena hasta el mes de agosto. Del total de casos atendidos, se reportaron que 354 fueron de violencia psicológica, 274 de violencia física, 76 de violencia sexual y 2 de violencia económica. Además, 578 de las víctimas fueron mujeres y 128 varones, y 217 casos las víctimas fueron adolescentes y menores de 14 años (5).

Ante lo ello la investigación tuvo como objetivo general determinar los tipos de violencia de género que más predomino en tiempos de COVID-19 en mujeres del Programa Vaso de Leche del Distrito de Huancavelica 2021.

\section{MATERIAL Y MÉTODOS}

El estudio fue de tipo descriptivo, observacional, retrospectivo, transversal. Esta investigación pretende "describir" los tipos de violencia de género que más predomino en tiempos de COVID-19 en mujeres del Programa Vaso de Leche del Distrito de Huancavelica 2021.

En la investigación se utilizó el diseño no experimental, transversal, descriptivo; ya que se realizó sin manipular la variable para recopilar datos e información para realizar una descripción, cuyo esquema es (6):

$$
\mathrm{M} \rightarrow \mathrm{O}
$$

Donde:

$\mathbf{M}=$ Muestra: Estuvo constituida por las mujeres con el cargo de presidentas del Programa Vaso de Leche del Distrito de Huancavelica 2021.

$\mathbf{O}=$ Observación: Violencia de género en tiempos de COVID-19.

La población de la investigación estuvo constituida por 47 mujeres con el cargo de presidentas del Programa de Vaso de Leche del Distrito de Huancavelica 2021. 
En el estudio la muestra fue censal, ya que se trabajó con toda la población de mujeres con el cargo de presidentas del Programa Vaso de Leche del Distrito de Huancavelica 2021.

El estudio no necesitó del muestreo puesto que en esta investigación se consideró a toda la población de estudio (censal).

La técnica que se utilizó en la investigación es la encuesta, consiste en recopilar información sobre una parte de la población denominada muestra.

Se utilizó el cuestionario que estuvo constituida por 25 ítems, 5 fueron los datos generales, 12 ítems sobre los tipos de violencia y 8 ítems sobre los efectos de la violencia; lo que permitió medir la variable del objeto de estudio, fue elaborado en base a la violencia de género y validado por un juicio de expertos.

\section{RESULTADOS}

En el presente estudio, se incluyó al total de presidentas del programa vaso de leche del distrito del Huancavelica del 2021.

Del 100,0\% (47 mujeres) de mujeres entrevistadas, fueron víctimas de violencia psicológica el 65,9\% (31 mujeres), económica 40,4\% (19 mujeres), física $31,9 \%$ (15 mujeres) y sexual el $21,3 \%$ (10 mujeres) (gráfico 1).
Tabla 1. Características sociodemográficas de las mujeres del programa vaso de leche del distrito de Huancavelica 2021.

\begin{tabular}{lccc} 
Edad & $\mathbf{F i}$ & $\mathbf{\%}$ & $\mathbf{\%}$ acumulado \\
18 a 29 & 12 & 25,5 & 25,5 \\
30 a 50 & 35 & 74,5 & 100,0 \\
Instrucción & $\mathbf{F i}$ & $\mathbf{\%}$ & $\mathbf{\%}$ acumulado \\
Primaria & 8 & 17,0 & 17,0 \\
Secundaria & 23 & 48,9 & 65,9 \\
Sup. Técnico & 11 & 23,5 & 89,4 \\
Sup. Universitario & 5 & 10,6 & 100,0 \\
Estado civil & $\mathbf{F i}$ & $\mathbf{\%}$ & $\mathbf{\%}$ acumulado \\
Conviviente & 29 & 61,7 & 61,7 \\
Casada & 10 & 21,3 & 83,0 \\
Soltera & 7 & 14,9 & 97,9 \\
Viuda & 1 & 2,1 & 100,0 \\
Ocupación & $\mathbf{F i}$ & $\mathbf{\%}$ & $\mathbf{\%}$ acumulado \\
Ama de casa & 39 & 83,0 & 83,0 \\
Trabajo independiente & 5 & 10,6 & 93,6 \\
Estudiante & 2 & 4,3 & 97,9 \\
Empleada pública & 1 & 2,1 & 100,0 \\
Procedencia & $\mathbf{F i}$ & $\mathbf{\%}$ & $\mathbf{\%}$ acumulado \\
Urbana & 30 & 63,8 & 63,8 \\
Rural & 17 & 36,2 & 100,0 \\
Total & $\mathbf{4 7}$ & $\mathbf{1 0 0 , 0}$ & \\
\hline
\end{tabular}

Fuente: Cuestionario sobre violencia de género en tiempos de COVID-19 en mujeres del Programa Vaso de Leche del distrito de Huancavelica 2021.

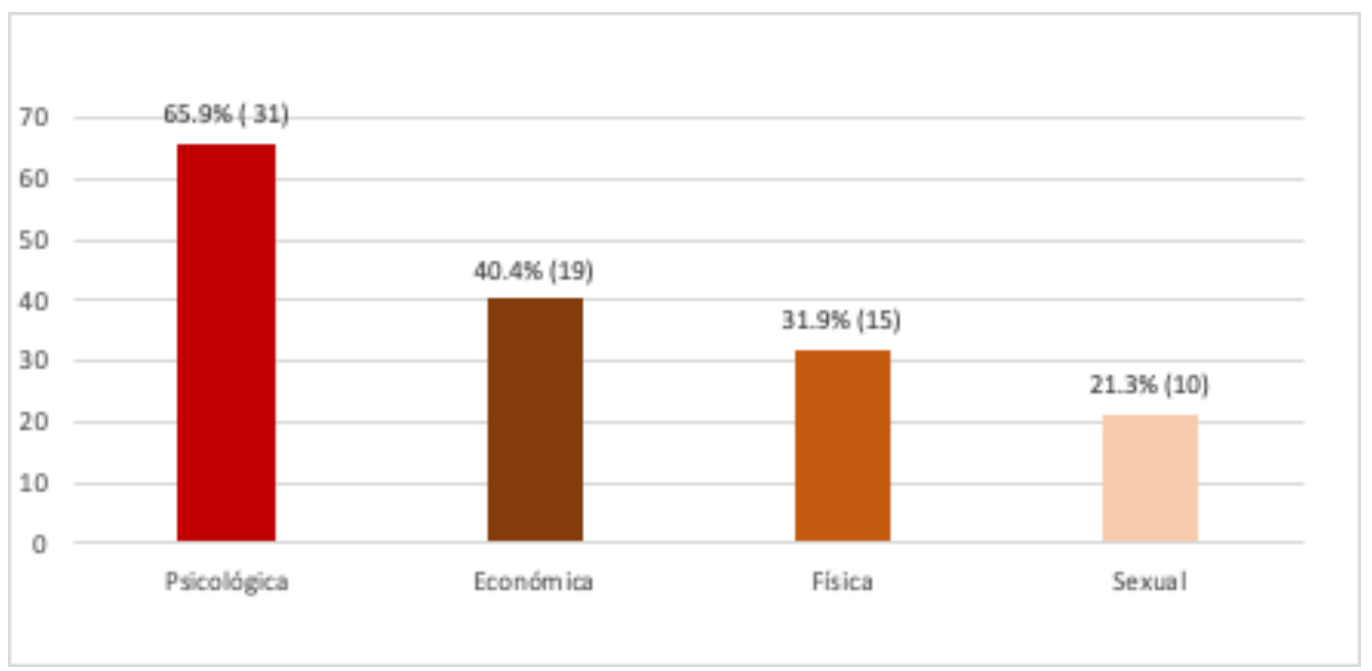

Fuente: Cuestionario sobre violencia de género en tiempos de COVID-19 en mujeres del Programa Vaso de Leche del distrito de Huancavelica 2021.

Gráfico 1. Tipos de violencia de género que más predomino en tiempos de covid-19 en mujeres del programa vaso de leche del distrito de Huancavelica 2021. 
Del 100,0\% (47 mujeres) de mujeres entrevistadas, tienen de 18 a 29 años $25,5 \%$ (12 mujere) y de 30 a 50 años $74,5 \%$ (35 mujeres); instrucción primaria 17,0\% ( 8 mujeres), secundaria 48,9\% (23 mujeres), superior técnico $23,5 \%$ (11 mujeres) y superior universitario $10,6 \%$ (5 mujeres); estado civil conviviente $61,7 \%$ (29 mujeres), casada $21,3 \%$ (10 mujeres), soltera $14,9 \%$ (7 mujeres) y viuda $2,1 \%$ (1 mujer); ocupación de ama de casa $83,0 \%$ (39 mujeres), trabajadora independiente $10,6 \%$ (5 mujeres), estudiante $4,3 \%$ ( 2 mujeres) y empleada publica $2,1 \%$ (1 mujer); de procedencia urbana $63,8 \%$ (30 mujeres) y rural $36,2 \%$ (17 mujeres) (tabla 1).

Indica que de 47 mujeres entrevistadas el 100,0\% presentaron efectos ante la violencia psicológica, así como también, ansiedad y depresión 61,9\% (26 mujeres) y baja autoestima 38,1\% (16 mujeres) (tabla 2).

Quince mujeres presentaron efectos ante la violencia física como: hematomas $46,7 \%$ (7 mujeres) y lesiones, heridas y/o fracturas en cualquier parte de su cuerpo en el 53,3\% (8 mujeres) (tabla 3 ).

De las 47 mujeres entrevistadas 10 mujeres presentaron efectos ante la violencia sexual como:

Tabla 2. Efectos que ocasiona la violencia psicológica en las mujeres del programa vaso de leche del distrito de Huancavelica 2021.

\begin{tabular}{lcc}
\hline Efecto & Fi & $\mathbf{\%}$ \\
\hline Ansiedad y depresión & 26 & 61,9 \\
Baja autoestima & 16 & 38,1 \\
Total & $\mathbf{4 2}$ & $\mathbf{1 0 0 , 0}$
\end{tabular}

Fuente: Cuestionario sobre violencia de género en tiempos de COVID-19 en mujeres del Programa Vaso de Leche del distrito de Huancavelica 2021.

Tabla 3. Efectos que ocasiona la violencia física en mujeres del programa vaso de leche del distrito de Huancavelica 2021.

\begin{tabular}{lll}
\hline Efecto & $\mathbf{F i}$ & $\mathbf{\%}$ \\
\hline Hematomas & 7 & 46,7 \\
Lesiones, heridas, ... & 8 & 53,3 \\
Total & $\mathbf{1 5}$ & $\mathbf{1 0 0 , 0}$ \\
\hline
\end{tabular}

Fuente: Cuestionario sobre violencia de género en tiempos de COVID-19 en mujeres del Programa Vaso de Leche del distrito de Huancavelica 2021. embarazo no deseado $20,0 \%$ ( 2 mujeres) y disminución del deseo sexual 80,0\% (8 mujeres) (tabla 4 ).

De 47 mujeres entrevistadas, 31 mujeres presentaron efectos ante la violencia económica como: Bajo ingreso económico 58,1\% (18 mujeres) y perdida de oportunidad educativa, laboral o social 41,9\% (13 mujeres) (tabla 5).

Tabla 4. Efectos que ocasiona la violencia sexual en mujeres del programa vaso de leche del distrito de Huancavelica 2021.

\begin{tabular}{lcc}
\hline Efecto & Fi & \% \\
\hline Embarazo no deseado & 2 & 20,0 \\
Disminución del deseo sexual & 8 & 80,0 \\
Total & $\mathbf{1 0}$ & $\mathbf{1 0 0 , 0}$ \\
\hline
\end{tabular}

Fuente: Cuestionario sobre violencia de género en tiempos de COVID-19 en mujeres del Programa Vaso de Leche del distrito de Huancavelica 2021.

Tabla 5. Efectos que ocasiona la violencia económica en mujeres del programa vaso de leche del distrito de Huancavelica 2021.

\begin{tabular}{lcc}
\hline Efecto & Fi & \% \\
\hline Bajo ingreso económico & 18 & 58,1 \\
$\begin{array}{l}\text { Perdida de oportunidad educativa, } \\
\text { laboral o social }\end{array}$ & 13 & 41,9 \\
Total & $\mathbf{3 1}$ & $\mathbf{1 0 0 , 0}$ \\
\hline
\end{tabular}

Fuente: Cuestionario sobre violencia de género en tiempos de COVID-19 en mujeres del Programa Vaso de Leche del distrito de Huancavelica 2021.

\section{DISCUSIÓN}

La presente investigación nos permite apreciar los resultados sobre la incidencia de los tipos de violencia de género en mujeres presidentas del programa vaso de leche del Distrito de Huancavelica. Este estudio fue realizado en una población y muestra de 47 presidentas, encontrándose así que el 65,9\% sufrieron de violencia psicológica, el 40,4\% sufrieron violencia económica, el $31,9 \%$ sufrieron violencia física y el $21,3 \%$ sufrieron de violencia sexual; en donde es evidente que la violencia psicología tuvo mayor incidencia, resultado que concuerdan con lo reportado por Matassini et al., encontró en su investigación que la violencia psicología tiene mayor porcentaje 
con un 49\% (7), también coincide con los resultados reportados por Romero en su investigación encontró que el 95\% sufrieron de violencia psicológica haciéndolo poner en el primer lugar de los diferentes tipos de violencia (8). Sin embargo los resultados difieren con lo hallado por Castillo et al., , donde se encontró que en su investigación el tipo de violencia que más predomino fue la violencia física con un $38,2 \%$ (9); estas similitudes y diferencias se deben a que las investigaciones se realizaron en diferentes contextos y poblaciones.

Las características demográficas de la mujeres presidentas del programa vaso de leche del Distrito de Huancavelica son: Respecto al grupo etario, el $25,5 \%$ son jóvenes y el 74,5\% son adultos; al grado de instrucción, el 17,0\% son de nivel primaria, el $48,9 \%$ de nivel secundaria, el $23,5 \%$ de nivel superior técnico y el 10,6\% de nivel superior universitario; al estado civil son conviviente el $61,7 \%$, casada el $21,3 \%$, soltera $14,9 \%$ y viuda $2,1 \%$; respecto a la ocupación el $83,0 \%$ son ama de casa, trabajadora independiente $10,6 \%$ y otros $6,4 \%$; y por ultimo respecto a lugar de procedencia urbana $63,8 \%$ y rural $36,2 \%$.

Esta investigación reporta que del total de mujeres víctimas de violencia psicológica, el 61,9\% sufrieron de ansiedad y depresión, y un 38,1\% sufrieron de baja autoestima, resultado que coincide con lo reportado por Mulato y Poma en su investigación reporta que el $100 \%$ de las víctimas de la violencia psicología presentaron tristeza, depresión o angustia, el $66.7 \%$ presentaron extrema falta de confianza (baja autoestima) (10); también coincide con los resultados reportados por Castillo et al., donde en su investigación menciona que este tipo de agresión repercute en la salud psicológica de la mujer (9).

Con respecto a los efectos de la violencia física, se encontró que del total de víctimas de este tipo de violencia (15); el 46,7\% presentaron hematomas y el $53,3 \%$ presentaron lesiones, heridas y/o fracturas en cualquier parte de su cuerpo, resultado que discrepan con lo reportado por Mulato \& Poma, donde en su investigación se encontró que las consecuencias de la violencia física en las mujeres fueron: $46,2 \%$ presentaron hematomas, $25,6 \%$ presentaron laceraciones (10).

En cuanto a los efectos de la violencia sexual, se encontró que del total de víctimas de este tipo de violencia (10); el 20,0\% presentaron embarazo no deseado y el $80,0 \%$ presentaron una disminución del deseo sexual. Respecto a lo mencionado no se encontró investigaciones similares a nivel internacional, nacional y local; para corroborar lo resultados hallados. Esta investigación halló los efectos de la violencia económica, de 31 mujeres víctimas de esta violencia; el 58,1\% sufrieron un Bajo ingreso económico y el $41,9 \%$ sufrieron una pérdida de las oportunidades educativas, laborales o sociales. Respecto a lo mencionado no se encontró investigaciones similares a nivel internacional, nacional y local; para corroborar lo resultados hallados.

Los resultados nos muestran claramente que el tipo de violencia de género que más predomino fue la psicológica seguido de la económica, teniendo múltiples consecuencias.

\section{CONCLUSIONES}

Los tipos de violencia que más predomino en tiempos de la COVID-19 en las mujeres presidentas del programa vaso de leche fueron: La violencia psicológica tuvo mayor incidencia con un $65,9 \%$, seguido de la violencia económica con un 40,4\%.

En cuanto a las características sociodemográficas de las mujeres presidentas del programa vaso de leche del distrito de Huancavelica, respecto al grupo etario predominó el adulto joven con un $74,5 \%$, respecto al grado de instrucción predominó el nivel secundario con un $48,9 \%$, con respecto al estado civil predominó conviviente con un $61,7 \%$, respecto a la ocupación predominó ama de casa con un $83,0 \%$ y por último respecto al lugar de procedencia predominó la procedencia urbana con un $63,8 \%$.

Los efectos de la violencia psicológica que presentaron las 42 víctimas fueron: ansiedad y depresión un $61,9 \%$ y baja autoestima un $38,1 \%$.

Los efectos de la violencia física que presentaron las 15 víctimas fueron: hematomas un $46,7 \%$ y lesiones, heridas $\mathrm{y} / \mathrm{o}$ fracturas en cualquier parte de su cuerpo un $53,3 \%$.

Los efectos de la violencia sexual que presentaron las 10 víctimas fueron: embarazos no deseados $20,0 \%$ y disminución del deseo sexual 80,0\%.

Los efectos de la violencia económica que presentaron las 31victimas fueron: bajo ingreso económico un $58,1 \%$ y perdida de oportunidad educativa, laboral o social un $41,9 \%$. 


\section{REFERENCIAS BIBLIOGRAFICAS}

1. Comisión de Derechos Humanos de las Naciones Unidas. Eliminación de la violencia contra la mujer. Washington DC: Comisión de Derechos Humanos de las Naciones Unidas; 20 de abril de 2000.

2. Lorente-Acosta M. Violencia de género en tiempos de pandemia y confinamiento. Rev Esp Med Leg. 2020;46(3):139-45.

3. Organización Mundial de la Salud. Violencia contra la mujer. Ginebra: Organización Mundial de la Salud; 2017. (Citado el 21 de octubre de 2020). Disponible en: https://www.who.int/es/news-room/fact-sheets/ detail/violence-against-women

4. World Health Organization. Nuevo coronavirus Japón (procedente de China). Ginebra: World Health Organization; 2020. (Citado el 21 de octubre de 2020). Disponible en: http://www.who.int/csr/ don/17-january-2020-novel-coronavirus-japan-exchina/es/

5. Andina. MIMP: Línea 100 atendió 67,712 llamadas por abuso sexual y violencia en cuarentena. Lima: Agencia Andina; 2020. (Citado el 21 de octubre de 2020). Disponible en: https://andina.pe/agencia/ noticia-mimp-linea-100-atendio-67712-llamadasabuso-sexual-y-violencia-cuarentena-804367.aspx
6. Ríos RR. Metodología para la investigación y redacción. 1 ra Edición. España: Servicios Académicos Intercontinentales SL; 2019.

7. Matassini S, Duffoó M, Alvarez, V. Violencia de género/familiar en tiempos de cuarentena. Instituto de Evaluacion de Tecnologis en Salud e Investigacion; 2020.p.1-14.

8. Romero MV. Violencia de género: Tipo, fase y factores asociados en los distritos de Tumán y José Leonardo Ortíz del departamento de Lambayeque agosto - octubre 2015. Tesis de Grado. Chiclayo: Universidad San Martin de Porres; 2017.

9. Castillo E, Bernardo JV, Medina MA. Violencia de género y autoestima de mujeres del centro poblado Huanja - Huaraz, 2017. Horiz Méd Lima. 2018;18(2):47-52.

10. Mulato M, Poma SF. Violencia basada en género en las mujeres del centro de salud San CristóbalHuancavelica 2017.Tesis de Grado. Huancavelica: Universidad Nacional de Huancavelica; 2019.

Recibido: 18/01/2021

Aceptado: 14/05/2021 